Bio - grafía. Escritos sobre la Biología y su Enseñanza. ISSN 2027-1034

Edición Extraordinaria. p.p. 538 - 547

Memorias del IX Encuentro Nacional de Experiencias en Enseñanza de la Biología y la Educación Ambiental. IV Congreso Nacional de Investigación en Enseñanza de la Biología.

\title{
EL AGUA, MOTOR DE LA VIDA. UNA UNID AD DIDÁCTICA INNOVADORA COMO MEDIO DE TRANSMISIÓN EFICIENTE DE LOS VALORES NATURALES
}

\author{
WATER, MOTOR OF LIFE. AN INNOVATIVE TEACHING UNIT AS A MEANS OF \\ EFFICIENT TRANSMISSION OF NATURAL VALUES
}

\author{
Nuria Álvaro Mora ${ }^{1}$ \\ Jose Reig Armiñana ${ }^{2}$ \\ Olga Mayoral García-Berlanga ${ }^{3}$
}

\section{RESUMEN}

Nuestro modelo de desarrollo, insostenible, genera presiones que cambian el estado del medio ambiente. Surgen así problemas ambientales que han sido y siguen siendo objeto de preocupación en numerosas conferencias y declaraciones internacionales. En busca de soluciones a estos problemas es considerada la educación ambiental, eje central de la presente propuesta. Centrándonos en su dimensión pedagógica, concretamente la educación reglada, y con el propósito de contribuir a sus principios, se desarrolla una propuesta de unidad didáctica de carácter ambiental dirigida a estudiantes de 11 y 12 años y titulada "Agua, motor de la vida" que surge de un estudio preliminar realizado como Trabajo Final en el grado universitario de las Ciencias Ambientales (Universidad de Valencia, España). Esta unidad, tras su ejecución en la práctica docente, ha mostrado la adquisición de aprendizaje significativo de los/as estudiantes sobre la hidrosfera; la importancia y la problemática del agua a través del seguimiento de una metodología constructivista, considerada como la más apropiada para educar para la sostenibilidad.

PALABRAS CLAVE: Educación Ambiental, Hidrosfera, Unidad Didáctica, propuesta didáctica

\section{ABSTRACT}

Our unsustainable development model generates pressures that change the state of the environment. There are environmental problems that have been and continue to be a concern in many international conferences and declarations. In search of solutions to these problems, environmental education has been well considered and it is the central axis of our proposal. Focusing on its educational dimension, formal education, and in order to contribute to its principles, an environmental teaching unit is developed. It is addressed to 11-12 years old students and entitled "Water, motor of life" that arises from a preliminary study realized as end-of-degree project in the university degree of

\footnotetext{
${ }^{1}$ Estudiante de doctorado del Departamen to de Didáctica de las Ciencias Experimentales y Sociales de la Facultad de Magisterio de la Universidad de Valencia (España)

2 Profesor Asociado del Departamento de Didáctica de las Ciencias Experimentales y Sociales de la Facultad de Magisterio de la Universidad de Valencia

${ }^{3}$ Universitat de Valencia, España.
} 
Bio - grafía. Escritos sobre la Biología y su Enseñanza. ISSN 2027-1034

Edición Extraordinaria. p.p. 538 - 547

Memorias del IX Encuentro Nacional de Experiencias en Enseñanza de la Biología y

la Educación Ambiental. IV Congreso Nacional de Investigación en Enseñanza de la

Biología.

Environmental Sciences (university of Valencia, Spain). This unit, after its execution in the teaching practice, has shown the acquisition of significant learning of students on the hydrosphere; the importance and the problematic of the water by following a constructivist methodology, considered as the most appropriate to educate for sustainability.

KEYWORDS: Environmental Education, Hydrosphere, Teaching Unit

\section{INTRODUCCIÓN}

La educación ambiental, a pesar de ser una educación necesaria y respaldada por numerosos congresos y declaraciones internacionales, se ve obstaculizada por una sociedad en su mayoría exenta todavía del conocimiento de los problemas ambientales, de la conciencia de los mismos, y/o de iniciativa que les impulse a actuar en la búsqueda de su solución (Vilches, 2010).

A fin de contribuir a esta educación para el desarrollo sostenible (Novo, 2009), se hace aquí una propuesta educativa que consiste en un modelo de unidad didáctica de contenido ambiental y de índole innovador. Esta propuesta se centra en la dimensión pedagógica de la educación ambiental que implica el desarrollo de esta educación en la escuela y la transmisión de sus principios, valores y actitudes al alumnado que deberá tomar parte de la misma en muchas decisiones y actuaciones. En los centros escolares, los valores del propio profesorado, la estructura organizativa del centro, y en lo referente al alumnado, el conflicto de valores que provocan los agentes educativos no formales (familia, medios de comunicación...), confieren complejidad a la dimensión de desarrollo curricular al tener lugar un "conflicto de intereses y valores" (Pardo, 1995). Por ello, el ambiente de la escuela resulta crucial para un aprendizaje global y hace relevante dentro de él la inclusión de programaciones didácticas ambientales.

Dentro de la educación reglada, hacemos una propuesta de unidad didáctica dirigida a estudiantes de 11-12 años. Se ha querido enfatizar por su carácter ambiental el estudio de la hidrosfera por lo que la unidad didáctica tiene por título "El agua, motor de la vida". No obstante, podrían abordarse otros componentes y problemáticas de relevancia socio-ambiental siguiendo sus mismas estrategias de aprendizaje. Centrada no sólo en el aprendizaje significativo de los/as estudiantes a partir de la transmisión de principios, valores y actitudes de carácter ambiental, esta unidad, que surge de un estudio preliminar (Álvaro \& Reig, 2014), considera en su planteamiento los problemas actuales en la formación (Pardo, 1995): la falta de objetivos claros, la divergencia entre las actuaciones y los problemas reales, la dificultad de la integración de ese carácter multidisciplinar en su estructura y la correcta adaptación a las características del destinatario.

La aplicación de la presente propuesta de unidad en un centro de educación valenciano ha demostrado un mayor aprendizaje de los/as estudiantes que de seguirse unidades didácticas tradicionales (se aumentó la nota media de los estudiantes un $25 \%$ aproximadamente). Por ello, desarrollamos aquí los objetivos, los 
Bio - grafía. Escritos sobre la Biología y su Enseñanza. ISSN 2027-1034

Edición Extraordinaria. p.p. 538 - 547

Memorias del IX Encuentro Nacional de Experiencias en Enseñanza de la Biología y la Educación Ambiental. IV Congreso Nacional de Investigación en Enseñanza de la Biología.

contenidos, las estrategias, las actividades y las competencias de nuestra propuesta que son necesarias trabajar a fin de acercar al alumnado al conocimiento de estudio no sólo como concepto teórico, sino práctico y de promover su pensamiento crítico. También destacamos el seguimiento de una metodología constructivista necesaria para pasar de una actitud de los/as estudiantes pasiva, en la que los conocimientos son meramente aprendidos, a una actitud más activa que relacione los conceptos teóricos con la práctica.

\section{METODOLOGÍA}

En las II Jornadas de Educación Ambiental (en Valsaín, 1987), fue definida la metodología constructivista como el modelo didáctico más coherente con los principios y objetivos de la educación ambiental. A diferencias de otros modelos didácticos, esta metodología otorga valor al contexto además del conocimiento, tomando la idea de la complejidad; esto es considerar el medio físico y natural junto con el social y cultural. Esencial en esta metodología es la unión de los/as estudiantes con el objeto de conocimiento y la relevancia del suceso en la comprensión de la realidad como así alegan estudios al afirmar que "el conocimiento no es el resultado de una mera copia de la realidad, sino de un proceso dinámico e interactivo a través del cual la información es interpretada y reinterpretada" (Gómez-Granell \& Coll Salvador, 1994). Además del estudio del entorno complejo, la metodología constructivista tiene en cuenta la complejidad del sujeto.

Con el uso de esta metodología todos los contenidos de esta unidad se transmitirán, ofreciendo al alumnado herramientas que les permitan resolver una situación problemática a partir de crear sus propios procedimientos. Esto hará que el aprendizaje sea un proceso dinámico en el que se generan unas ideas, que con el aprendizaje van modificándose y generando, a su vez, nuevas ideas. La explicación de los contenidos será resultado de las respuestas de los/as estudiantes a las preguntas que se les planteen y que se realizarán en relación al contenido objeto de estudio. Para llevar a cabo la presente metodología y poder obtener un aprendizaje significativo, en la unidad "Agua, motor de la vida" se hace uso de unas estrategias que hacen efectiva esta propuesta y cuya principal finalidad es hacer reflexionar al alumnado, acercarlo a la realidad y hacerle partícipe del conocimiento (Tabla 1):

Tabla 1: Estrategias a utilizar en las unidades didácticas de estudio y objeto de las mismas.

\begin{tabular}{|l|l|}
\hline \multicolumn{1}{|c|}{ Estrategia } & \multicolumn{1}{|c|}{ Finalidad } \\
\hline Trabajo cooperativo & $\begin{array}{l}\text { Dinamización y reflexión conjunta. } \\
\text { Aprendizaje entre iguales }\end{array}$ \\
\hline Preguntas abiertas y de relacionar & Dinamización y reflexión \\
\hline $\begin{array}{l}\text { Trabajo bibliográfico, búsqueda de } \\
\text { información }\end{array}$ & Participación en el conocimiento \\
\hline Práctica de laboratorio & Acercamiento a la realidad \\
\hline Análisis de tablas, informes, gráficas, & Reflexión \\
\hline
\end{tabular}


Bio - grafía. Escritos sobre la Biología y su Enseñanza. ISSN 2027-1034

Edición Extraordinaria. p.p. 538 - 547

Memorias del IX Encuentro Nacional de Experiencias en Enseñanza de la Biología y la Educación Ambiental. IV Congreso Nacional de Investigación en Enseñanza de la Biología.

\begin{tabular}{|l|l|}
\hline imágenes y viñetas & \\
\hline Planteamiento de dilemas morales & Reflexión \\
\hline $\begin{array}{l}\text { Uso de competencias ajenas al medio } \\
\text { ambiente }\end{array}$ & $\begin{array}{l}\text { Visión de lo complejo y conocimiento de } \\
\text { formas de comunicación }\end{array}$ \\
\hline Observaciones de la realidad & Acercar la realidad \\
\hline
\end{tabular}

\section{Desarrollo de la unidad didáctica "agua, motor de la vida"}

La unidad didáctica se desarrolla en 6 sesiones de 50 minutos. Sus objetivos quedan concretados en la Tabla 2 junto con los contenidos, las actividades, los recursos educativos y las competencias evaluables que se trabajan en cada sesión.

Los objetivos de la unidad se centran en el conocimiento de la hidrosfera, la identificación de la problemática del agua y la justificación de la necesidad de su gestión sostenible. Se hace así un recorrido al alumnado que va desde el conocimiento del problema del objeto de estudio hasta la promoción de soluciones sostenibles, necesarias si se quiere educar para la sostenibilidad.

Por lo que se refiere a los contenidos, estos se han clasificado de acuerdo a si se tratan de contenidos conceptuales $(C)$, procedimentales $(P)$ o actitudinales $(A)$. En esta unidad didáctica se van a trabajar conceptos relacionados con el agua, se transmitirán contenidos siguiendo procedimientos de laboratorio y prácticas de aula y se hará una valoración de la importancia del agua que fomente sobre todo la generación de actitudes en los/as estudiantes.

Las actividades, por su parte, se han dividido en actividades de identificación de modelos previos (AIMP) enfocadas en la obtención de los conocimientos previos de los/as estudiantes, actividades de desarrollo (AD) que son las actividades que desarrollan la unidad y las actividades de ampliación y recopilación (AAYR) que sintetizan, conectan y amplían los diferentes contenidos trabajados. Cabe matizar que deberá elegir una de entre todas las actividades de ampliación (A28-A32) que se presentarán de manera atractiva haciendo uso de la Webquest, "actividad orientada a la investigación donde toda o casi toda la información que se utiliza procede de recursos de la Web" (Bernie Dodge, 1995, en Argote, Palomo, Sánchez \& Ruiz, 2009).

Con el desarrollo de esta unidad didáctica, los/as estudiantes adquirirán competencias que hemos clasificado de acuerdo a las fijadas en el anexo I 1105/2014 del Real Decreto, de 26 de diciembre, por el que se establece el currículo básico de la Educación Secundaria Obligatoria y del Bachillerato que regula la educación en España (Ministerio de Educación, Cultura y Deporte, 2015). Cabe resaltar la presencia en esta unidad de todas las competencias que establece el currículo español. Se muestran a continuación ejemplificadas con actividades de la unidad:

1. Competencia en comunicación lingüística (CCLI): Presentaciones orales, desarrollo de trabajos escritos, comunicación intrapersonal.

2. Competencia matemática y competencias básicas en ciencia y tecnología (CMCT) : Fomento del razonamiento científico en la resolución de problemas. 
Bio - grafía. Escritos sobre la Biología y su Enseñanza. ISSN 2027-1034

Edición Extraordinaria. p.p. 538 - 547

Memorias del IX Encuentro Nacional de Experiencias en Enseñanza de la Biología y la Educación Ambiental. IV Congreso Nacional de Investigación en Enseñanza de la Biología.

3. Competencia digital (CD) : Desarrollo de actividades de búsqueda y obtención de información en internet.

4. Aprender a aprender (CAA): Trabajo por grupos cooperativos, fomento de la iniciativa en el aprendizaje, valoración de la participación en el aprendizaje de la clase.

5. Competencias sociales y cívicas (CSC): Fomento del respeto a los compañeros, aplicación práctica de los conocimientos adquiridos en clase, enfoque de las actividades desde la comprensión de la realidad que nos rodea.

6. Sentido de iniciativa y espíritu emprendedor (SIEE) : Fomento del espíritu crítico e iniciativa en el desarrollo de las actividades.

7. Conciencia y expresiones culturales (CEC): Valoración de la capacidad estética y creadora de las presentaciones. Fomento del respeto a las distintas manifestaciones sobre la herencia cultural y trabajo de la imaginación en algunas actividades.

Tabla 2: Unidad didáctica "Agua, motor de la vida". Sus objetivos, contenidos, actividades, recursos didácticos y competencias evaluables.

\begin{tabular}{|c|c|c|c|c|c|}
\hline \multirow{4}{*}{$\stackrel{9}{9}$} & \multicolumn{5}{|c|}{$\begin{array}{l}\text { - Describir las características, composición y propiedades de la hidrosfera, } \\
\text { relacionándolas con la existencia de vida en la Tierra. }\end{array}$} \\
\hline & \multicolumn{5}{|c|}{$\begin{array}{l}\text { - Interpretar y reconocer el papel que juega el ciclo del agua, en la distribución } \\
\text { del agua en el planeta Tierra y reflexionar acerca de su uso y distribución según } \\
\text { los diferentes países. }\end{array}$} \\
\hline & \multirow{2}{*}{\multicolumn{5}{|c|}{$\begin{array}{l}\text { - Conocer e identificar los problemas de contaminación de las aguas actuales, } \\
\text { su origen y sus repercusiones. Reflexionar sobre ellos y generar posibles } \\
\text { soluciones con el fin de desenrollar actitudes y hábitos de protección del medio } \\
\text { ambiente. } \\
\text { - Justificar la necesidad de una gestión sostenible del agua y de actuaciones } \\
\text { personales y colectivas que potencian un uso responsable y la reducción de su } \\
\text { consumo. }\end{array}$}} \\
\hline & & & & & \\
\hline \multicolumn{2}{|c|}{ Sesión } & Contenidos & Actividades & Recursos & $\begin{array}{l}\text { Ompetencias } \\
\text { evaluables }\end{array}$ \\
\hline
\end{tabular}


Bio - grafía. Escritos sobre la Biología y su Enseñanza. ISSN 2027-1034

Edición Extraordinaria. p.p. 538 - 547

Memorias del IX Encuentro Nacional de Experiencias en Enseñanza de la Biología y la Educación Ambiental. IV Congreso Nacional de Investigación en Enseñanza de la Biología.

\begin{tabular}{|c|c|c|c|c|}
\hline 1 & ¡El agua, es & $\begin{array}{l}\text { AlMP: A.1. ¿Qué sabemos? } \\
\text { Anotemos en el cuaderno de } \\
\text { clase el vocabulario que } \\
\text { sabemos sobre la hidrosfera y } \\
\text { qué preguntas nos hacemos. } \\
\text { A.2. ¿Qué podemos decir de } \\
\text { un cubito de hielo? A3. } \\
\text { Experimenta en el laboratorio. } \\
\text { ¿Se calientan más los océanos } \\
\text { o los continentes? } \\
\text { AD: A4. Tan simple como } \\
\text { algo cotidiano. Relaciona } \\
\text { fenómenos cotidianos con } \\
\text { propiedades del agua. }\end{array}$ & $\begin{array}{l}\text { Grupo, } \\
\text { laboratorio } \\
\text { (recipientes } \\
\text { iguales, } \\
\text { arena, } \\
\text { termómetros, } \\
\text { foco) }\end{array}$ & - \\
\hline 2 & $\begin{array}{l}\text { La hidrosfera } \\
\qquad(\mathrm{C}) \\
\text { Propiedades. } \\
\text { El agua en la } \\
\text { Tierra (P) }\end{array}$ & $\begin{array}{l}\text { AD: A.5. El agua en granitos } \\
\text { de arroz. Representemos a } \\
\text { través de } 100 \text { granos de arroz, } \\
\text { el porcentaje que refleja la } \\
\text { distribución de las aguas en el } \\
\text { planeta y digamos cuántos } \\
\text { granos representan el agua que } \\
\text { usamos. A.6. } \\
\text { El agua es vida. ¿Por qué se } \\
\text { dice que el agua es vida? A.7. } \\
\text { El origen del agua. Veamos el } \\
\text { siguiente vídeo. A.8. ¿Dónde } \\
\text { está el agua? Hemos visto el } \\
\text { origen del agua y su importancia } \\
\text { pero, ¿Sabríais decirme dónde } \\
\text { podemos encontrar agua? A.9. } \\
\text { ¡En casa! En busca de la } \\
\text { mejor noticia. Busquemos y } \\
\text { hagamos un resumen crítico de } \\
\text { una noticia que trate los } \\
\text { aspectos trabajados en esta } \\
\text { sesión de clase. }\end{array}$ & $\begin{array}{c}\text { Arroz, } \\
\text { internet } \\
\text { (vídeo. } \\
\text { https://www.y } \\
\text { outube.com/w } \\
\text { atch?v=fPRjG } \\
\text { qmTY2M), } \\
\text { ordenadores, } \\
\text { calculadora, } \\
\text { grupo }\end{array}$ & $\begin{array}{c}\text { CD, CCLI, } \\
\text { CMCT, CAA, } \\
\text { SIEE }\end{array}$ \\
\hline 3 & $\frac{\frac{\text { ¿El agua }}{\text { realmente se }}}{\underline{\text { agota? }}}$ & $\begin{array}{l}\text { AD: A.10. Un dibujo de mi } \\
\text { realidad. El agua está en } \\
\text { continuo movimiento. ¿Podríais } \\
\text { dibujar el ciclo del agua? A.11. } \\
\text { Construcción de nuestro } \\
\text { modelo. Comparemos nuestros } \\
\text { dibujos con los de los } \\
\text { compañeros y completémoslo. } \\
\text { A.12. El ciclo del agua. A.13. } \\
\text { El ciclo urbano del agua. ¡Nos } \\
\text { falta el factor antrópico! Añadirlo }\end{array}$ & $\begin{array}{l}\text { Actividades } \\
\text { ciclo del } \\
\text { agua, } \\
\text { Internet } \\
\text { (http://concur } \\
\text { so.cnice.mec } \\
\text { es/cnice200 } \\
\text { 5/63_el_agua } \\
\text { /actividades/ } \\
\text { activ_explora } \\
\text { ndo_agua.ht }\end{array}$ & $\begin{array}{c}\text { CMCT, } \\
\text { SIEE, CCLI, } \\
\text { CAA, CSC }\end{array}$ \\
\hline
\end{tabular}


Bio - grafía. Escritos sobre la Biología y su Enseñanza. ISSN 2027-1034

Edición Extraordinaria. p.p. 538 - 547

Memorias del IX Encuentro Nacional de Experiencias en Enseñanza de la Biología y la Educación Ambiental. IV Congreso Nacional de Investigación en Enseñanza de la Biología.

\begin{tabular}{|c|c|c|c|c|}
\hline & & $\begin{array}{l}\text { al dibujo tras interactuar con él } \\
\text { en internet. A.14. ¡En casa! El } \\
\text { paisaje del agua: Agua } \\
\text { continental y marina. El agua } \\
\text { conforma paisajes muy } \\
\text { diferentes. Encuentra una } \\
\text { imagen o haz tú mismo una } \\
\text { fotografía a un paisaje } \\
\text { continental y otro marino y } \\
\text { cuélgalo en las redes. }\end{array}$ & $\begin{array}{l}\text { ml), grupo, } \\
\text { folios A3 }\end{array}$ & \\
\hline 4 & $\frac{\frac{\text { ¿Qué }}{\text { funciones }}}{\underline{\text { tiene el }}}$ & 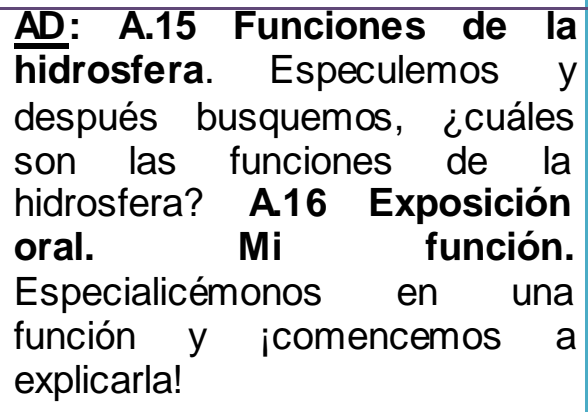 & $\begin{array}{l}\text { Ordenadores, } \\
\text { internet }\end{array}$ & $\begin{array}{c}\text { CMCT, } \\
\text { CCLI, CAA }\end{array}$ \\
\hline 5 & $\begin{array}{l}\text { de la } \\
\text { importancia } \\
\text { para los } \\
\text { seres vivos y } \\
\text { para la } \\
\text { calidad de } \\
\text { vida }(A)\end{array}$ & $\begin{array}{l}\text { AD: A.17. ¿Para qué uso el } \\
\text { agua? Observad las siguientes } \\
\text { imágenes y averiguad para que } \\
\text { usamos el agua. A. 18. Lectura: } \\
\text { Agua que mata. A. 19. iEn } \\
\text { casa! Reflexión acerca de la } \\
\text { importancia del agua. Tras lo } \\
\text { estudiado en clase, ¿podrías } \\
\text { decirme qué importancia tiene el } \\
\text { agua? }\end{array}$ & $\begin{array}{c}\text { Imágenes } \\
\text { uso del agua, } \\
\text { fotocopias } \\
\text { lectura } \\
\text { (http://elpais.c } \\
\text { om/diario/200 } \\
\text { 9/08/02/socie } \\
\text { dad/1249164 } \\
002 \text { _850215. } \\
\text { html) }\end{array}$ & $\begin{array}{l}\text { CCLI, CAA, } \\
\text { CSC, CEC }\end{array}$ \\
\hline 6 & $\begin{array}{c}\frac{\text { ¿Cómo es la }}{\text { realidad? }} \\
\begin{array}{c}\text { Contaminació } \\
\mathrm{n}(\mathrm{P})\end{array}\end{array}$ & $\begin{array}{l}\text { AD: A.20. Mapa conceptual: La } \\
\text { contaminación del agua } \\
\text { continental. ¿Qué grupo creará } \\
\text { el mapa conceptual más } \\
\text { completo? A21. Mapa } \\
\text { conceptual: La contaminación } \\
\text { del mar. ¿Qué grupo creará el } \\
\text { mapa conceptual más } \\
\text { completo? A22. ¡En casa! } \\
\text { ¡Menuda contaminación! }\end{array}$ & $\begin{array}{c}\text { Fichas para } \\
\text { el mapa } \\
\text { conceptual de } \\
\text { la } \\
\text { contaminació } \\
\text { n, actividades } \\
\text { prácticas } \\
\text { sobre } \\
\text { contaminació } \\
\text { n }\end{array}$ & $\begin{array}{c}\text { CMCT, } \\
\text { CEC, CCLI, } \\
\text { CSC, CAA }\end{array}$ \\
\hline
\end{tabular}


Bio - grafía. Escritos sobre la Biología y su Enseñanza. ISSN 2027-1034

Edición Extraordinaria. p.p. 538 - 547

Memorias del IX Encuentro Nacional de Experiencias en Enseñanza de la Biología y la Educación Ambiental. IV Congreso Nacional de Investigación en Enseñanza de la Biología.

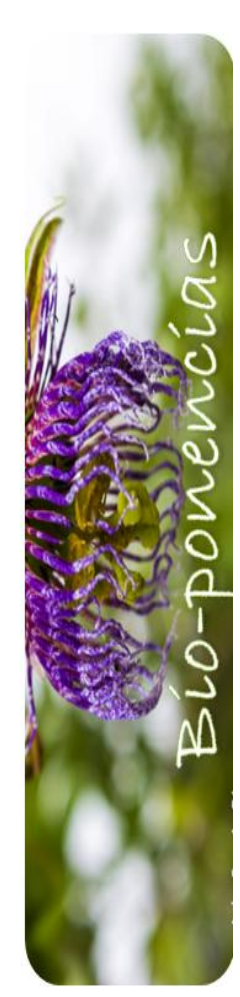

\begin{tabular}{|c|c|c|}
\hline 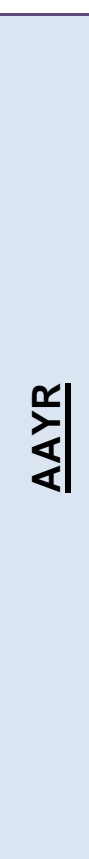 & $\begin{array}{l}\text { A.25. Ejercita tu memoria. } \\
\text { (http://www.testeando.es/asignatura.asp?idC=7\&idA=11). } \\
\text { A.26. Elaborar mapa conceptual de la unidad. A.27. } \\
\text { Recordando las propiedades del agua (propiedades, } \\
\text { características y ejemplos). A.28. La hidrosfera y su relación } \\
\text { con el cambio climático. Todos conocemos sobre el cambio } \\
\text { climático. ¿Puede que éste tenga efectos sobre la hidrosfera? } \\
\text { Averígualo. A.29. Análisis de la factura del agua. Cada dos } \\
\text { meses en casa se paga la factura del agua. Pero, ¿qué es?, } \\
\text { ¿cómo se interpreta?, ¿qué significa? ¡Dínoslo! } \\
\text { A.30. Y yo, ¿qué puedo hacer? La degradación de las aguas } \\
\text { es un hecho presente hoy en día. La pregunta es ¿qué podemos } \\
\text { hacer nosotros para evitarla? A.31. Las mareas negras. Los } \\
\text { vertidos de petróleo tienen efecto en las aguas marinas. Estas } \\
\text { aguas presentan múltiples funciones por los que los efectos se } \\
\text { expanden más allá de las mismas. Averigua lo que sepas sobre } \\
\text { las mareas negras A.32. Las corrientes marinas. ¿Qué son? } \\
\text { Pero sobre todo, ¿por qué son importantes? }\end{array}$ & $\begin{array}{l}\text { CMCT, CD, } \\
\text { SIEE, CCLI, } \\
\text { CAA, CSC, } \\
\text { CEC }\end{array}$ \\
\hline
\end{tabular}

\section{Evaluación de la intervención}

Tradicionalmente, la evaluación se ha centrado en la etapa final del aprendizaje y se ha concebido, de forma general, para aprobar más que para aprender. En esta propuesta educativa se propone una evaluación continua para facilitar la asimilación y el desarrollo progresivos de los contenidos aportados al alumnado y de las competencias que deben alcanzarse. Además, este tipo de evaluación ofrece al profesorado un mayor y mejor seguimiento del progreso en el aprendizaje del estudiante, ya que permite una valoración integral (Delgado \& Oliver, 2006).

La adquisición de competencias por los/as estudiantes, hace que sea relevante una evaluación por competencias. Aunque todas las actividades dan a conocer al docente y al alumnado el aprendizaje resultante, proponemos calificar por su peso dentro de la unidad didáctica las siguientes: A. 9, A. 12, A. 14, A. 16, A. 19, A. 22 y A. Ampliación (la escogida entre todas las que se exponen). Dicha calificación vendrá dada tras establecer unos criterios de evaluación para cada una de las competencias evaluables. A continuación se muestra algunos criterios para la competencia matemática y competencias básicas en ciencia y tecnología (Tabla 3). Consideramos que cada docente es quien debe establecer los criterios de acuerdo a las características de su alumnado y contexto.

Con estos criterios se evaluará la adquisición por parte de los/as estudiantes de cada una de las competencias trabajadas en las actividades calificables. El cumplimiento de todos los criterios de evaluación dentro de una misma competencia supondrá el alcance de 5 puntos. Por tanto, se hará uso de la escala del 1 al 5 , en función del cumplimiento por los/as estudiantes de los criterios de evaluación (de menor a mayor cumplimiento, respectivamente). 
Bio - grafía. Escritos sobre la Biología y su Enseñanza. ISSN 2027-1034

Edición Extraordinaria. p.p. 538 - 547

Memorias del IX Encuentro Nacional de Experiencias en Enseñanza de la Biología y la Educación Ambiental. IV Congreso Nacional de Investigación en Enseñanza de la Biología.

Tabla 3: Criterios de evaluación ejemplificativos para la competencia matemática y competencias básicas en ciencia y tecnología.

\section{Competencia matemática y competencias básicas en ciencia y tecnología (CMCT):}

- Operaciones básicas

- Resolución de problemas

- Expresión del razonamiento

- Comprensión del conocimiento/identifica cuestiones

- Adquisición destrezas científicas/Argumentar/Explicar

- Aplicación conocimiento/uso de pruebas/modelización

\section{CONCLUSIÓN}

Nuestro desarrollo actual que no contempla los costes ambientales nos está conduciendo a un estado ambiental degradado que requiere la puesta en marcha de soluciones. El alcance de un desarrollo sostenible demanda la formación de una ciudadanía consciente de la importancia del pilar ambiental en el nuevo desarrollo y de su función dentro del mismo.

Hablamos de educar para el desarrollo sostenible. Concienciar al alumnado en las buenas prácticas ambientales y permitirle formar parte de ese proceso a favor del desarrollo sostenible. Se requiere llevar a cabo programaciones didácticas de índole ambiental en la escuela, pues en ella se desarrolla el proceso educativo, pero sin dejar de lado la educación no reglada, fundamental también dado el carácter multidisciplinar de la educación ambiental.

Nuestra tarea se ha centrado en el desarrollo de una propuesta de unidad didáctica como modelo en el abordaje de problemáticas de relevancia socio-ambiental. Titulada "Agua, motor de la vida", se centra en el tema de la hidrosfera con aplicación a estudiantes de 11-12 años y tiene por metodología la constructivista. Dado su carácter ambiental se han propuesto actividades y seguido estrategias enfocadas en el alcance de un aprendizaje significativo de los/as estudiantes con el fin de promover actitudes de respeto al medio ambiente. La unidad didáctica propuesta aporta conocimiento sobre la hidrosfera, concretamente el uso actual del agua así como su importancia en la vida y su problemática con el fin de, en primer lugar, conocer los hechos objetivamente, y seguidamente reflexionar, crear ideas, construir opiniones críticas hasta actuar en dirección a esa opinión construida.

El desarrollo de una buena programación didáctica basada en la interiorización de conocimientos y valores naturales que además pretende un aprendizaje significativo, 
Bio - grafía. Escritos sobre la Biología y su Enseñanza. ISSN 2027-1034

Edición Extraordinaria. p.p. 538 - 547

Memorias del IX Encuentro Nacional de Experiencias en Enseñanza de la Biología y la Educación Ambiental. IV Congreso Nacional de Investigación en Enseñanza de la Biología.

supone una buena manera de convertir "La Hidrosfera" en "Agua, motor de la vida", lo que a su vez supone no sólo escuchar, olvidar o recordar, sino también aprender, comprender y actuar. Porque para prevenir los problemas ambientales se hace necesario una ciudadanía concienciada, dispuesta y participativa.

\section{BIBLIOGRAFÍA}

Álvaro, N., \& Reig, P. (2014). Desarrollo de las unidades didácticas para la Educación secundaria y bachillerato relacionados con la Educación Ambiental. Una propuesta innovadora. (Trabajo final de grado). Universidad de Valencia, Facultad de Ciencias biológicas, España.

Argote, J., Palomo, R., Sánchez, J., \& Ruiz, J. (2009) Concepto y elementos de una WQWebquest. WQWebquest un recurso educativo para su uso en el aula. Málaga: Universidad de Málaga. Recuperado de: http://tecnologiaedu.uma.es/materiales/wq/archivos/cap1 WQ Definicion.pdf

Delgado, A.M., \& Oliver, R. (2006). La evaluación continua en un Nuevo escenario docente. Revista de Universidad y Sociedad del Conocimiento 3(1) pp.1-11. Recuperado de: http://www.uoc.edu/rusc/3/1/dt/esp/delgado oliver.pdf

Gómez-Granell, C., \& Coll Salvador, C. (1994). De qué hablamos cuando hablamos de constructivismo. Cuadernos de pedagogía (221), pp. 8-10.

Ministerio de Educación, Cultura y Deporte (2015). Real Decreto 1105/2014, de 26 de diciembre, por el que se establece el currículo básico de la Educación Secundaria Obligatoria y del Bachillerato. Recuperado de: http://www.boe.es/boe/dias/2015/01/03/pdfs/BOE-A-2015-37.pdf

Novo, M. (2009). La educación ambiental, una genuina educación para el desarrollo sostenible. Revista de Educación, (número extraordinario), pp. 195-21. Recuperado de:

http://www.revistaeducacion.mec.es/re2009/re2009 09.pdf

Pardo, A. (1995). La Educación Ambiental como proyecto. España: Institut de Ciències de l'Educació. Universitat de Barcelona

Vilches, A., Gil, D. y Cañal, P. (2010). Educación para la sostenibilidad y educación ambiental. Investigación en la Escuela, 71, pp. 5-15. 